\title{
Ewingella americana
}

National Cancer Institute

\section{Source}

National Cancer Institute. Ewingella americana. NCI Thesaurus. Code C86391.

A species of facultatively anaerobic, Gram negative, rod shaped bacterium assigned to the phylum Proteobacteria. This species is oxidase neg ative, fermentative, susceptible to ampicillin, amoxicillin-clavulanate, ceftazidime, ceftriaxone, cefotaxime, cefepime, ofloxacin, gentamicin, carbenicillin, and amikacin but resistant to cephalothin, penicillin G, and vancomycin. A rare human pathogen, it can be isolated from human blood, stool, urine, wounds, conjunctiva, respiratory tract, and peritoneal dialysate of immunocompromised patients. 